\title{
X-RAY SPECTROSCOPY TO DETERMINE LINE COINCIDENCES \\ BETWEEN R- AND L-SHELL TRANSITIONS
}

\author{
P.G. Burkhalter, D. Newman*, J.V. Gilfrich and D.B. Brown \\ Naval Research Laboratory \\ Washington, DC 20375 \\ P.D. Rockett and-G. Charatis \\ KMS Fusion, Inc. \\ Ann Arbor, MI 48106 \\ C. Hailey and D.L. Matthews \\ Lawrence Livermore National Laboratory \\ Livermore, CA 94550 \\ B. MacGowan \\ Imperial College \\ London, England
}

Accurate wavelengths for highly-ionized L-shell spectra were measured in the $10-16 \AA$ region. The purpose being to determine lines in coincldence with L-shell transitions from the elements oxygen, fluorine, and neon. L-shell transitions have been proposed for resonant photopumping of $\mathrm{K}$-shell electrons in these elements t.9) generate lasing between upper levels in the $40-150 \mathrm{eV}$ region. (T) The current effort improves on and expands the earlier spectroscopic work performed at KMS Fusion, Inc., where possible line coincidences werk identified for photolonizing in the 1-3 and 1-4 levels in fluorine. New experimental techniques have led to a wavelength accuracy now believed to be $+2 \mathrm{~m} \AA$ for cases in which adequate calibration lines are available. Exact spectral line matches were found for $\mathrm{Mn}$ with the $\mathrm{F} \mathrm{H}_{\text {- }}$ line at $12.643 \AA$ and for both $\mathrm{Mn}$ and $\mathrm{Cr}$ with the $\mathrm{F} \mathrm{He}, \mathrm{H}^{\prime}$ line at 14.458\%." The Mn line at $12.643 \AA$ has been ideqtified, using ab initio atomic structure calculations, as the $\mathrm{D}_{2}-\mathrm{F}_{3}$ transition in Be-like Mn XXII. The Mn line emissivity was determined to be $30 \mathrm{MW}$ into 2 steradians for a conversion efficiency of $0.04 \%$. Photopumping with Mn coated gasfilled tajgets is presently being tried in gain measurement experiments at LLNL.

$\mathrm{X}$-ray spectra were collected under controlled illumination and target conditions in order to examine a number of potential L-shell lines. These lines represented cases where near agreement between L-shell transitions and the 1-3 transitions in He- or H-like $0, F$ and Ne had been previously reported. The KMSF CHROMA laser was used to generate a plasma source of soft x-ray emission. The glass laser was operated in the frequency-doubled modq $4(0.537 \ldots \mathrm{m})$ for enhanced light absorption at an irradiance of $1.5 \cdot 10^{14} \mathrm{~W} / \mathrm{cm}^{2}$.

Several new techniques were used in this experiment to improve the wavelength accuracy. These included (1) the use of a cylindricallyfocused lens and end-on viewing of the plasma source, (2) the use of short-laser pulses of $120 \mathrm{psec}$ duration to reduce the extent of $x-r a y$ (*Sachs/Freeman Associates, Inc., Bowie, MD 20715) 
emission due to plasma expansion, (3) the targets were viewed at $90^{\circ}$ to the laser beam to minimize the Doppler shifts, (4) the use of highsensitivity $x$-ray film and active-element intensified spectrographs, and (5) in a few shots, a split target was used to confirm the line coincidences in single laser shots.

Two high-resolution spectrographs viewed the target in the same plane, both at $90^{\circ}$ to the laser beam. This arrangement allowed viewing the plasma end-on, resulting in an effective source size of about $100 \mathrm{~m}$. Another intensified spectrograph viewed from behind the target. Its diffraction crystal was set to record spectral data at wavelengths corresponding to the 1-2 transitions in the ionized gases.

Spectral data were collected directly onto film in the passive spectrograph. the film selected was Kodak direct exposure film 50445 (DEF). This film has a good sensitivity and a low background fog level. The PEF film is about 3 times more sensitive than Kodak No Screen film. (4) Spectral data were recorded in either one or two shots per element with the passive spectrograph, and with intensified spectrographs incorporating microchannel plates.

The data were recorded as spectral pairs on the spectrograms. Target elements for calibration purposes were carefully selected for each of the spectral regions investigated. Fig. I shows a typical spectrogram collected with a beryl diffracting crystal directly onto film. The 2p-3d F-1ike Fe XVIII transitions were used for calibration of the Mn lines. Fig. 2 shows spectrograms collected with one of the intensified spectrographs near $12.6 \AA$. Line callbration for the $\mathrm{Mn}$ spectra was provided by $\mathrm{Fe}, \mathrm{Ca}$, and $\mathrm{Cr}$ lines in this spectral region. These juxapositioned spectral pairs were read on a Grant comparator densitometer which was set up to read both strips alternately with a Decker system. In a few shots, confirmation of the line coincidences was observed in spectrograms from split targets. These targets were formed by coating half the plastic substrate with the candidate element and the other half with $\mathrm{CaF}_{2}$. By using spatial imaging, the upper spectra in Fig. 2 were collected in a single laser shot.

The results of the wavelength measurements are listed in the Table for the various spectral regions. Exact Iine coincidences were found in the first two cases with fluorine 1-3 transitions. The wavelengths for the nearest lines are listed for other metal L-spectra that match $\mathrm{k}-11$ nes in neon and oxygen (in two cases within $4 \mathrm{~m} \AA$ ). Lines were absent in Mn spectra that coincided with $1-2$ transitions in fluorine. These would have spolled the lasing scheme by filling the lower level.

\section{References}

1. P.L. Hagelstein, Plasma Physics 25, 1345 (1983), and "Physics of Short Wavelength Laser Design," UCRL-53100 (1981).

2. P.G. Burkhalter, G. Charatis, and P.D. Rockett, J. App1. Phys. 54, 6138 (1983).

3. D.L. Matthews, et a1. (current experiments on Novette).

4. P.D. Rockett, etal. (to be published).

5. C. Hailey, et al. Second Topical Meeting on Laser Techniques in the Extreme Ultraviolet, Mar. 5-7, 1984, Boulder, Co. 

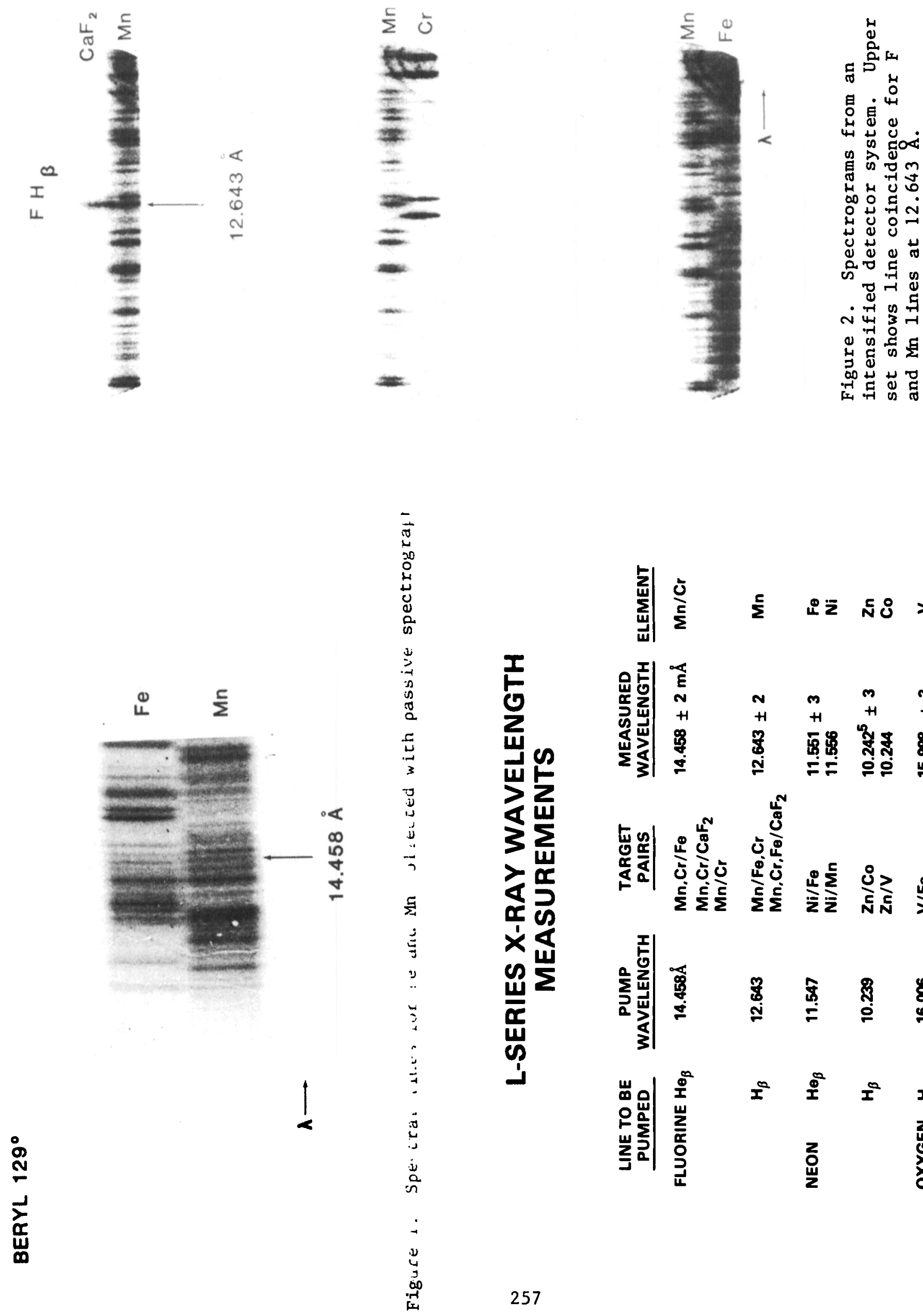

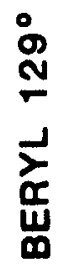
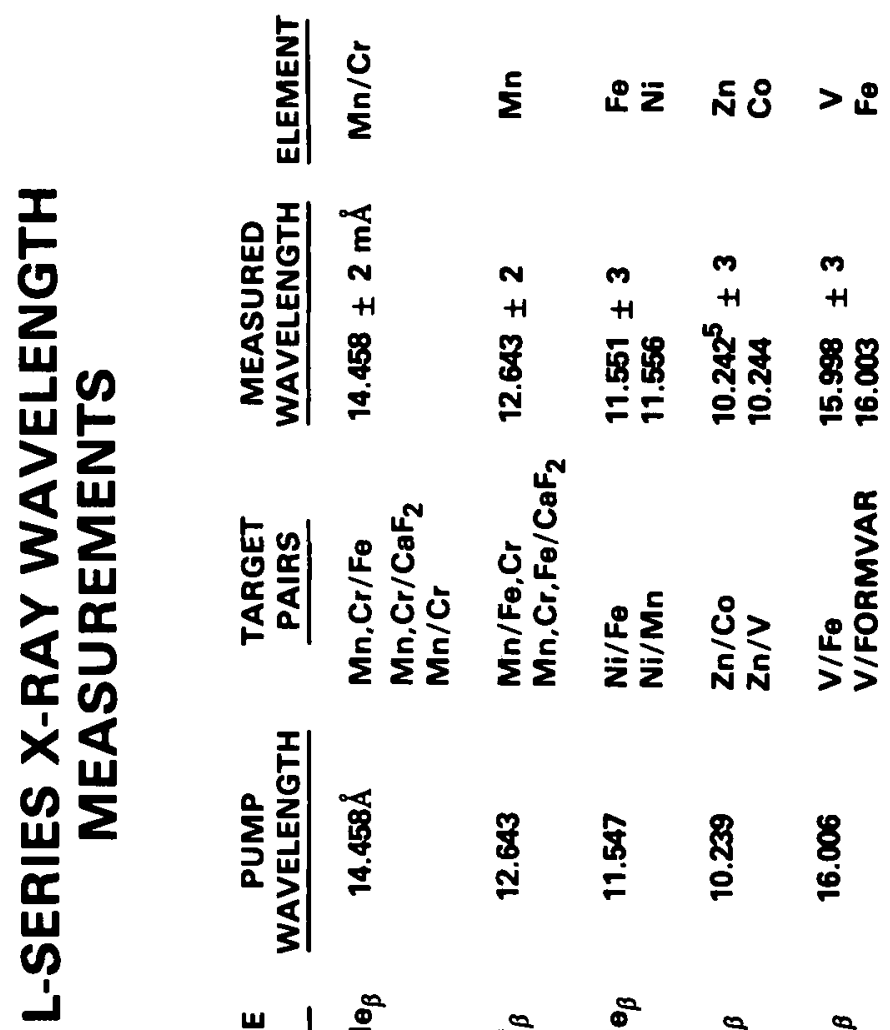

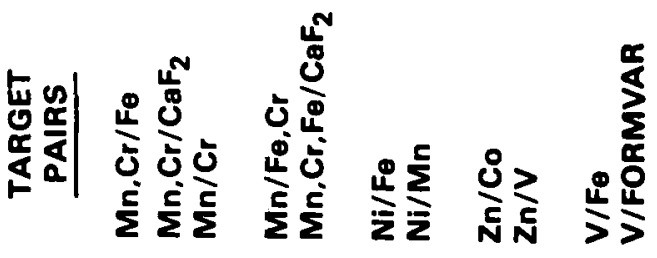
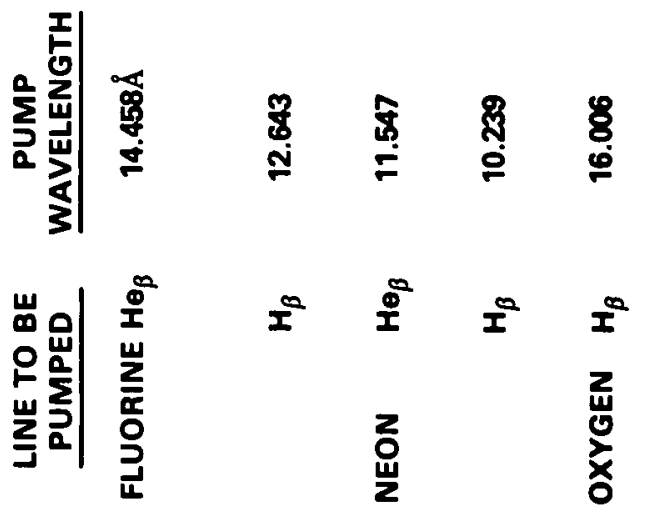